\title{
Use of a case-control study and geographic information systems to determine environmental and demographic risk factors for canine leptospirosis
}

\author{
George S. GHNEIM ${ }^{\mathrm{a}, \mathrm{b} *}$, Joshua H. VIERS ${ }^{\mathrm{c}}$, Bruno B. CHOMEL ${ }^{\mathrm{d}}$, \\ Philip H. KASS ${ }^{\mathrm{d}}$, Daphne A. DESCOLlONGES ${ }^{\mathrm{a}}$, Michael L. JOHNSON ${ }^{\mathrm{e}}$ \\ ${ }^{a}$ Center for Companion Animal Health, School of Veterinary Medicine, University of California, \\ Davis, CA 95616, USA \\ ${ }^{\mathrm{b}}$ RTI International, 3040 Cornwallis Rd, PO Box 12194, Research Triangle Park, NC, 27709-2194, USA \\ ${ }^{\mathrm{c}}$ Department of Environmental Science and Policy, University of California, Davis, CA 95616, USA \\ ${ }^{\mathrm{d}}$ Department of Population Health and Reproduction, School of Veterinary Medicine, \\ University of California, Davis, CA 95616, USA \\ ${ }^{\mathrm{e}}$ John Muir Institute of the Environment, University of California, Davis, CA 95616, USA
}

(Received 1 April 2006; accepted 19 July 2006)

\begin{abstract}
Leptospirosis is increasingly diagnosed as a re-emerging canine disease in the USA. Our objectives were to describe potential risk factors for canine leptospirosis infections in northern California, through the use of a case-control study, and to perform a spatial analysis to investigate which aspects of the landscape and land use patterns are important in the transmission of leptospirosis. Forty-three cases and 59 controls were enrolled into the study. Serological results showed that $17(39.5 \%)$ of the $43 \mathrm{dog}$ cases were infected with serovar pomona. Cases were 7.86 times more likely to have been walked in a rural environment rather than an urban environment. Cases also had eight times higher odds of swimming in outdoor water, and approximately 12 times higher odds of drinking from outdoor water in the two weeks preceding illness. At smaller distances from the dogs' homes (radius $\leq 0.5 \mathrm{~km}$ ) hydrographic density was positively correlated with cases, while at larger distances (radius $\geq 5 \mathrm{~km}$ ) there was a positive relationship between leptospirosis cases and percent of wetlands or public open space. Intervention measures for the prevention of canine leptospirosis should include reducing access to potentially infectious bodies of water that are close to canine homes, and to large areas of wetlands and public open space in the general vicinity. We have shown that a spatial analysis in conjunction with traditional epidemiological analysis is a powerful combination in identifying risk factors for infectious diseases.
\end{abstract}

leptospirosis / dogs / GIS / California / disease geography

\section{INTRODUCTION}

Leptospirosis is a widely distributed disease of humans, domestic animals, and wildlife caused by bacteria of the genus Leptospira. There are currently over 200

* Corresponding author: gsghneim@rti.org pathogenic serovars of this organism, and it has a worldwide distribution [8]. Leptospirosis has long been known as a disease of dogs, with Leptospira interrogans serovars canicola and icterohaemorrhagiae as the primary agents historically involved in canine infections. From the early 
1970 's to the early 1990 's there was a sharp decline in the incidence of canine leptospirosis throughout the USA [16]. This change was attributed to the commercial leptospirosis vaccine that was commonly used at that time, since that vaccine protected dogs against serovars canicola and icterohaemorrhagiae [16].

But the last decade has seen a reversal of this trend, and now leptospirosis is being increasingly diagnosed, and documented as a re-emerging canine disease nationwide in the USA $[1,3,22,23,25]$. Coinciding with the increase in incidence was a change of the infecting serovars. In recent studies dogs were primarily infected with three serovars: bratislava, grippotyphosa, and pomona, which were not included in historic canine vaccines. It should also be noted that most of the diagnoses were made based on serology, primarily the microscopic agglutination test (MAT), and that cross-reactivity among serovars is a common occurrence [3].

We know that leptospirosis is intricately tied to fresh water, and human or canine outbreaks have usually followed heavy rainfall or flooding [7,21]. But it is still unclear what led to the recent increase in incidence of canine leptospirosis and more specifically the change in infecting serovars. Leptospira bacteria are shed in the urine of an infected individual and survive well only in a moist and $\mathrm{pH}$ neutral environment [7]. Infection is spread when another animal's mucous membranes or disrupted epithelium come into contact with contaminated water [7]. While rodents are generally considered the primary reservoir for leptospirosis, cattle and small mammals such as raccoons, opossums, and skunks are often implicated as other important reservoirs [16, 17]. The diagnostic challenges associated with this disease make monitoring incidence difficult and only with time consuming and costly active surveillance can we accurately assess the incidence of leptospirosis. Dogs are good sentinels for detecting the presence of Leptospira in the environment and are key factors to understanding the ecology of this disease. Because of their importance as pets, many dogs benefit from aggressive diagnostics and treatment which has enabled clinicians and researchers to gain insight into the current status of leptospirosis in many geographic areas.

Our primary objective was to describe the potential risk factors associated with canine leptospirosis infections in northern California, through the use of a retrospective case-control study of dogs that were presented at the Veterinary Medical Teaching Hospital (VMTH) at the School of Veterinary Medicine, University of California, Davis, USA.

Our secondary objective was to perform a spatial analysis to investigate which aspects of the landscape and land use patterns are important in the transmission of leptospirosis. We used a Geographical Information System (GIS) as a tool to perform our spatial analyses. Recent applications of these spatial analytic techniques to vector borne diseases, such as Lyme disease [12], and arboviral diseases [15] have demonstrated the emerging utility of this technique.

\section{MATERIALS AND METHODS}

\subsection{Case-control study}

\subsubsection{Animals}

We performed a search in the VMTH database for any dog that was serologically tested for leptospirosis from January 1st 1998 to December 31st 2000. The MAT was the primary diagnostic test used at the VMTH. Not all canines included in the study had paired serum titers performed. Therefore, a case definition was developed that was applied to individuals that were sampled at least once. Dogs that had a 
single titer of 1:800 or greater without indication of vaccination for leptospirosis in the three months prior to illness were presumptively considered to be infected with Leptospira [3], and were enrolled as cases. All dogs that had been vaccinated in the three months before being examined at the VMTH were excluded, to prevent any misclassification. It was necessary to exclude these recently vaccinated dogs because we could not evaluate which vaccines the dogs had received from their regular veterinarian. Similarly, any dogs with titers of 1:400 or 1:200 (i.e. suspects) were excluded to ensure that the results were valid and to avoid misclassification. Since serological titers in response to leptospirosis infections can decrease rapidly, it is unclear if dogs with a titer of 1:200 or 1:400 were truly infected or just had a residual titer from past vaccinations [3]. Dogs that had been examined at the VMTH and had leptospirosis serology performed with resulting titers of 1:100 or less were enrolled as controls. The controls were generally dogs with acute renal disease, as the primary clinical sign in the most recent cases of leptospirosis was generally acute renal disease $[1,17,25]$. Due to the small sample size, we selected all the controls that were available, resulting in a 1 to 1.4 ratio of cases to controls, and we could not perform any matching. The serovar with the highest titer was reported as the serovar most likely to have caused the infection [4].

\subsubsection{Demographics}

All cases and controls were examined for any difference in demographic attributes that included county of residence, breed category, gender, and age. For dogs with two residences, we used the primary residence for county of residence. Counties were designated as either coastal or inland. Coastal counties were those defined as any county that bordered the ocean, bay, or delta water. Breeds were classified into the seven breed categories, as defined by the American Kennel Club Inc. (AKC), with an eighth category designated for all mixed breed dogs. These seven AKC categories are sporting dogs, terriers, working dogs, non-sporting dogs, herding dogs, hounds and toy dogs. Four gender categories were defined as intact male, neutered male, intact female, and neutered female. We defined age groups as less than one year, one to three years, four to seven years, and eight years or older.

\subsubsection{Questionnaire}

We developed a questionnaire that addressed various environmental issues that were potential risk factors for leptospirosis infection. The questionnaire included questions about 21 potential risk factors. The questions dealt with all aspects of a dog's environment that could lead to exposure to Leptospira organisms, e.g. exposure to water, being off leash, walking in an urban environment, and exposure to wildlife. Vaccine history was excluded because few owners knew if the Leptospira portion of the vaccine was included in their dog's most recent annual vaccination. We also included a question relating to whether the owners saw wildlife species such as rodents, raccoons, skunks, or opossums in the dog's environment. The questionnaire was administered to the owners of both cases and controls and referred to the two week period before the patients were seen at the VMTH.

\subsubsection{Data analysis}

We evaluated differences in exposure to demographic and environmental risk factors in cases versus controls using chisquare tests of homogeneity and computing odds ratios (OR) with $95 \%$ confidence 
intervals $(95 \% \mathrm{CI})$ and $P$-values. Those variables whose associated $P$-values were $<0.05$ were further analyzed using an unconditional logistic regression model. For each category of variables, all variable levels were compared to the first level (baseline) of the variable. Adjusted OR and $95 \% \mathrm{CI}$ were estimated using EGRET Statistical Software (c) 2001 Cytel Software Corp., Cambridge, MA, USA). Levels within variables for which there were no values were removed.

\subsection{GIS study}

\subsubsection{Map design}

Each case and control dog was identified for location by using Topo USA 3.0 (C) DeLorme, Yarmouth, ME, USA) address-matching software to determine a general latitude and longitude. We excluded cases and controls for which we could not obtain a home address. We also excluded three geographic outliers which had home addresses in Nevada (1) and the southern portion of California (2), since it was not reasonable to include these dogs in the same geographic analysis; furthermore, for dogs with more than one residence, we used the primary residence. A point coverage of case and control locations was generated and projected in ArcGIS 8.1 (Environmental Systems Research Institute, Redlands, CA, USA) to Albers Equal Area (Fig. 1), to match preexisting geospatial data. Initial results from the case-control portion of the study led us to focus on a suite of landscape scale factors to be analyzed within the GIS. These included hydrographic features (e.g. streams and rivers), forms of land use (e.g. urban, forest, agriculture, and wetland), and accessibility to open space.

We examined cases and controls as a nominal response variable against several landscape factors at varying scales within the GIS to (a) determine which factors are likely better predictors for coarse screening of disease carriers and (b) to determine at which scale these factors are likely more influential. We addressed the second objective by buffering each point using fixed radii of $0.5 \mathrm{~km}(78.5 \mathrm{ha})$, $2 \mathrm{~km}$ (1256.6 ha), $5 \mathrm{~km}$ (7854 ha), and $10 \mathrm{~km}$ (31415.9 ha). These circular areas, which we termed Levels of Spatial Influence (LSI), contain landscape features in different proportions (Fig. 2) allowing us to detect appropriate scales of influence by various landscape predictors. The four different radii represent proxies for different modes of exposure; LSI 1 to LSI 4 encompass a range of dog-owner behaviors, from off-leash near-home access, to neighborhood walks, to local park visitation, and to regional park access. For each LSI, we calculated landscape predictors from three separate sources of data: stream features, where we calculated hydrographic density (unit length/unit area LSI); percent managed open space (total area of managed open space/unit area LSI); and percent land use category (total area of land use/unit area LSI) using urban, forest, agriculture, and wetland categories.

Our stream features are defined by the National Hydrographic Dataset (NHD) ${ }^{1}$; which included streams, rivers, lake edges, and other similar edge feature types. Although our water features (e.g. ponds, streams) are effectively static, prevalent dynamic hydrological processes (e.g. floods) are inherently spatially coincident with the presence of pre-existing water features.

We focused on public open space, managed for multiple uses such as recreation

\footnotetext{
${ }^{1}$ USGS 2003, Standards for National Hydrography Dataset: National Mapping Program Technical Instructions, 1999, US Environmental Protection Agency, US Department of the Interior, US Geological Survey National Mapping Division, http://nhd.usgs.gov/.
} 


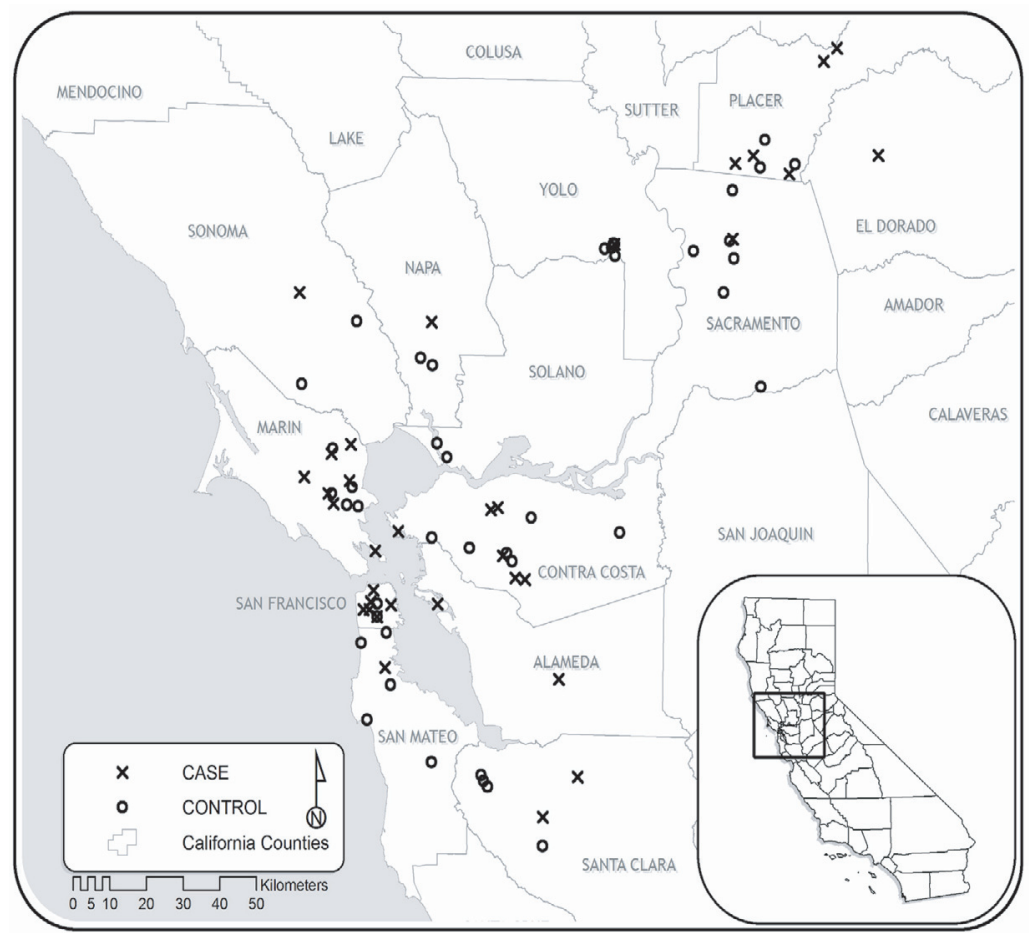

Figure 1. Geographical representation of canine leptospirosis cases and controls in northern California identified during the three year period, 1/1/1998 to 12/31/2000. (A color version of this figure is available at www.edpsciences.org/vetres.)

as a landscape factor. Open space, as defined for our study, and developed by others [2] includes the following: city, regional, state, and national parks; land trusts and sanctuaries; wilderness areas and nature reserves; recreation areas and beaches; as well as parcels designated as conservation easements. While not all such open spaces guarantee access to dogs, they do serve as preserved habitats for wildlife. We also focused on land uses, as defined and mapped by the National Land Cover Database (NLCD 2001) [10]. We isolated the following land uses: urban areas, characterized by $>30 \%$ constructed materials (e.g. asphalt, buildings, etc.); forested lands, characterized $>25 \%$ by tree cover; agriculture, defined as $>75 \%$ land planted or cultivated for food, feed, or fiber (note: we excluded urban grasses); and wetlands, which were identified using the saturated soil criteria defined by Cowardin [5].

\subsubsection{Statistical analyses}

We examined the overall geographic trend for any spatial biases by performing a bivariate logistic regression analysis of cases and controls as a function of latitude and longitude. We also examined the relationship between mean annual precipitation and disease status, by examining long term rainfall data [6]. We then performed a series of bivariate logistic regression analyses to examine factor relationships between case/control responses and hydrographic density, open space availability, and categorical land-uses for each 


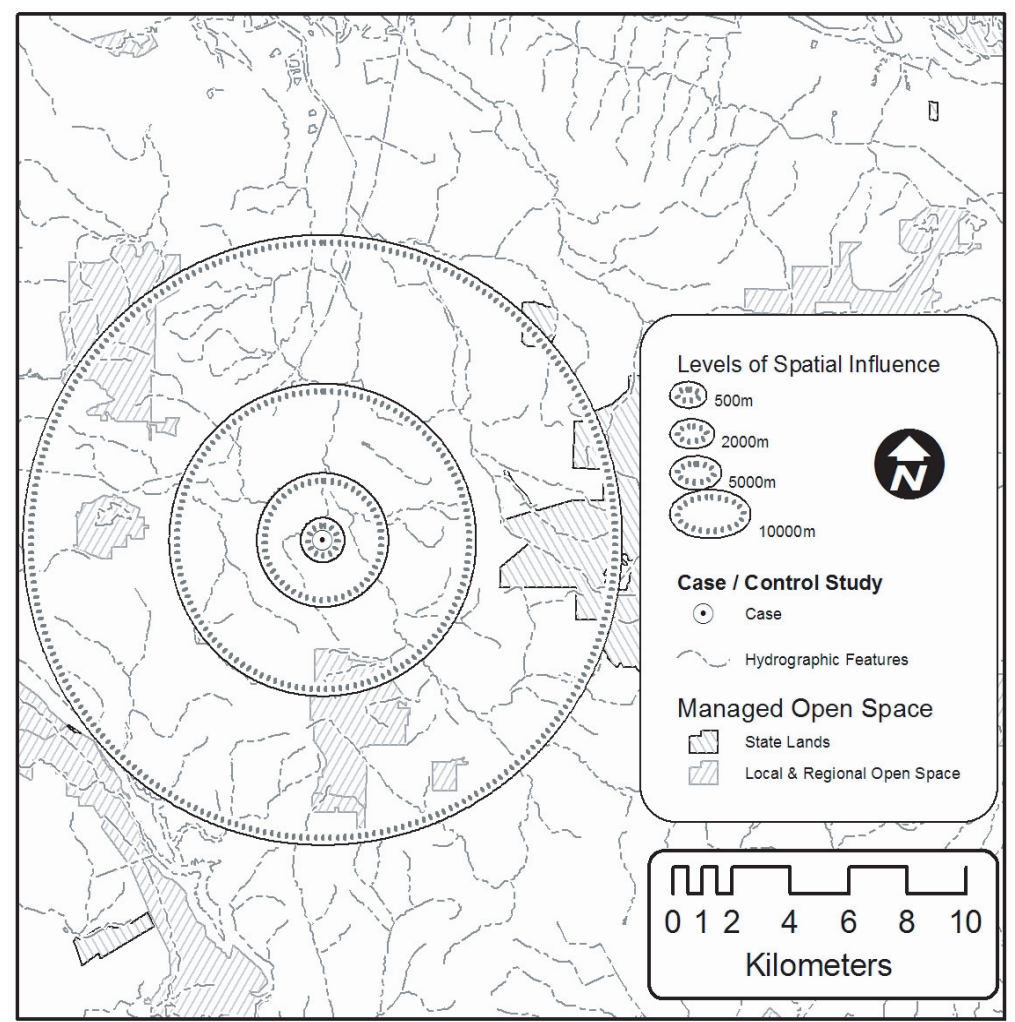

Figure 2. Example of a case/control with the four LSI, and types of managed open spaces. (A color version of this figure is available at www.edpsciences.org/vetres.)

LSI. We constructed multivariate models for each LSI using respective landscape factor values, selecting forward step-wise predictors $(P \leq 0.10)$ to determine relative scale of influence using Receiver Operating Characteristic (ROC) area-under-the-curve (AUC) for comparing model performance [18]. We then created a single, multi-scale multivariate nominal logistic regression model to see if similar landscape factors predominated across scales and across the study area. We used JMP (version 5.0) for all statistical analyses (SAS Institute, Cary, NC, USA) and examined our results for potential non-linear relationships by performing similar regressions with square and square root transformations of the independent variables. We also assessed linearity of our continuous predictor variables to avoid model misspecification by using a BoxTidwell transformation test with interaction terms in our full model. Lastly, in our full landscape model, we report likelihood ratio (LR) $\chi^{2}$ values of significance for the continuous independent factors, since the Wald statistic is prone to Type II errors [13].

\section{RESULTS}

\subsection{Case-control study}

\subsubsection{Animals}

We enrolled 43 cases and 59 controls into the study, since 28 individuals were 
Table I. Leptospira interrogans serovar(s) infections diagnosed by MAT.

\begin{tabular}{lc}
\hline $\begin{array}{l}\text { Serovar(s) with } \\
\text { highest titer }\end{array}$ & $\begin{array}{c}\text { Number Diagnosed } \\
(N=43)\end{array}$ \\
\hline pomona & $17(39.5 \%)$ \\
canicola & $4(9.3 \%)$ \\
bratislava & $2(4.7 \%)$ \\
grippotyphosa & $2(4.7 \%)$ \\
icterohaemorrhagiae & $2(4.7 \%)$ \\
hardjo & $1(2.3 \%)$ \\
pomonal & $11(25.6 \%)$ \\
bratislava & \\
grippotyphosa/ & $1(2.3 \%)$ \\
canicola & \\
pomona/bratislava/ & $2(4.7 \%)$ \\
icterohaemorrhagiae & \\
pomona/bratislaval & $1(2.3 \%)$ \\
grippotyphosa/canicola & \\
\hline
\end{tabular}

defined as suspects and thus not included in the study. Questionnaires were completed for only 30 cases $(70 \%)$ and 36 controls $(61 \%)$, due to a lack of current contact information or refusal. The serological results for the 43 cases demonstrated that $17(39.5 \%)$ dogs were infected with serovar pomona, four $(9.3 \%)$ were infected with serovar canicola, two dogs $(4.7 \%)$ were infected with serovar bratislava, two dogs $(4.7 \%)$ were infected with serovar grippotyphosa, two dogs $(4.7 \%)$ were infected with serovar icterohaemorrhagiae, one dog $(2.3 \%)$ was infected with serovar hardjo, and 15 dogs (34.9\%) had equal titers to more than one serovar (Tab. I).

\subsubsection{Unconditional multiple logistic regression model}

For the demographic risk factors, only age was statistically significant (Tab. II). Cases had significantly higher odds of being less than a year old or of being eight years or older when compared to dogs between one and three years of age. These results indicate that cases were approximately 16.5 times more likely to be juvenile dogs and approximately 11 times more likely to be older dogs than dogs between one and three years of age. The comparisons of intact males to all females, and coastal versus inland counties were borderline significant (Tab. II).

The analysis of the questionnaire responses indicated five significant environmental risk factors (Tab. III). Cases had 11.15 higher odds of living only in one residence rather than sharing their time between two residences. Cases were 7.86 times more likely to have been walked in a rural environment rather than an urban environment. Our examination of water sources or non-specific swimming activities did not lead to any significant risk factors. However, we determined that dogs that drank outdoor water or swam in outdoor water in the two weeks before presentation at the VMTH were at risk. Cases had eight times higher odds of swimming in outdoor water, and approximately 12 times higher odds of drinking from outdoor water in the two weeks preceding illness. The last significant risk factor was wild animal exposure, with cases having approximately five times higher odds of having a known exposure to wild animals (e.g., rodents, skunks, opossums, raccoons, or coyotes). Exposure to different categories of wild animals was not significant.

\subsection{GIS study}

Geographic location was useable for $36(84 \%)$ cases and $50(85 \%)$ controls (Fig. 1). Examination for geographic trends indicated no spatial correlation in latitude $(P=0.78)$. Longitude was significant $(P=0.035)$, but accounted for minimal variation in our observations $\left(r^{2}=\right.$ 0.038). Such longitudinal trends are of 
Table II. Case-control study of leptospirosis in dogs, VMTH, Jan 1, 1998-Dec 31 2000: Demographic risk factors, and logistic regression model results.

\begin{tabular}{lcccccc}
\hline Risk factor & Variables & Cases & Controls & Total & Odds ratio & $\begin{array}{c}95 \% \text { Confidence } \\
\text { interval }\end{array}$ \\
\hline County & Inland & 12 & 28 & 40 & 1 & NA \\
& Coastal & 28 & 29 & 57 & 2.25 & $(0.96,5.29)$ \\
Breed group & Mixed & 11 & 8 & 19 & 1 & NA \\
& Toy & 0 & 3 & 3 & 0.00 & $(0.00, \infty)$ \\
& Hound & 6 & 3 & 9 & 1.45 & $(0.28,7.64)$ \\
& Herding & 8 & 12 & 20 & 0.48 & $(0.14,1.74)$ \\
& Non-sporting & 0 & 10 & 10 & 0.00 & $(0.00, \infty)$ \\
& Working & 6 & 7 & 13 & 0.62 & $(0.15,2.58)$ \\
& Terrier & 1 & 4 & 5 & 0.18 & $(0.017,1.95)$ \\
Gender & Sporting & 11 & 12 & 23 & 0.67 & $(0.20,2.27)$ \\
& Intact females & 2 & 7 & 9 & 1 & NA \\
& Neutered females & 15 & 24 & 39 & 2.19 & $(0.40,11.96)$ \\
& Intact Males & 10 & 6 & 16 & 5.83 & $(0.90,37.82)$ \\
& Neutered males & 16 & 22 & 38 & 2.55 & $(0.47,13.91)$ \\
& All females & 17 & 31 & 48 & 1 & NA \\
& Intact males & 10 & 6 & 16 & 3.04 & $(0.94,9.81)$ \\
& & & & & & \\
& 1-3 years & 1 & 11 & 12 & 1 & NA \\
& $<\mathbf{1}$ year & $\mathbf{3}$ & $\mathbf{2}$ & $\mathbf{5}$ & $\mathbf{1 6 . 5 0}$ & $(\mathbf{1 . 0 9 , 2 5 0 . 2 0})$ \\
& 4-7 years & 16 & 24 & 40 & 7.33 & $(0.86,62.50)$ \\
$\geq \mathbf{8}$ years & $\mathbf{2 2}$ & $\mathbf{2 2}$ & $\mathbf{4 4}$ & $\mathbf{1 1 . 0 0}$ & $\mathbf{( 1 . 3 1 , 9 2 . 6 3 )}$ \\
\hline & & & & & &
\end{tabular}

possible concern because precipitation is positively correlated with longitude - due to the proximity of the Pacific Ocean to our study area ( $r=0.36 ; n=86$ case/controls) and to the state of California $(r=0.67 ; n=$ 1000 random sites). However, there was no detectable difference in mean annual precipitation (1961-1990 annual average) between cases and controls $(P=0.33)$. When observations greater than $-120.10{ }^{\circ} \mathrm{W}$ in longitude were excluded ( $n=5$ controls), the longitudinal trend was no longer present $(P=0.38)$. Furthermore, these easterly controls were significantly further from the geographic center of all observations than the other data $(P<0.0001$; mean difference $=164.9 \mathrm{~km}$ ) and were excluded from all subsequent analyses.
We analyzed cases as a positive response using logistic regression. Our initial screening results showed that log-distance to the nearest hydrographic feature was positively related to infection status $(P=$ 0.0006 , pseudo- $\left.r^{2}=0.11\right)$, indicating that the probability of infection increased the closer the dwelling address was to a hydrographic feature. However, we also wanted to determine which other factors, and at which scale of proximity, were acting to promote infection. To address this question, we constructed multivariate logistic regression models at each of our four levels of spatial influence using a suite of landscape factors.

We used forward stepwise nominal logistic regression to find the best model at 
Table III. Case-control study of leptospirosis in dogs, VMTH, Jan 1 1998-Dec 31 2000: Environmental risk factors with significant logistic regression model results.

\begin{tabular}{|c|c|c|c|c|c|c|}
\hline Risk factor & Variables & Cases & Controls & Total & Odds & $95 \%$ confidence \\
\hline & & & & & ratio & interval \\
\hline 2nd residence & Yes & 1 & 10 & 11 & 1.00 & NA \\
\hline & No & 29 & 26 & 55 & 11.15 & $(1.34,93.18)$ \\
\hline Where walked & Urban & 2 & 10 & 12 & 1.00 & NA \\
\hline & Rural & 11 & 7 & 18 & 7.86 & $(1.31,47.05)$ \\
\hline & Remote (wild) & 14 & 15 & 29 & 4.67 & $(0.87,25.14)$ \\
\hline Swam 2 weeks before illness & No & 18 & 32 & 50 & 1.00 & NA \\
\hline & Yes & 9 & 2 & 11 & 8.00 & $(1.56,41.14)$ \\
\hline Drank outdoor water 2 weeks before illness & No & 9 & 28 & 37 & 1.00 & NA \\
\hline & Yes & 16 & 4 & 20 & 12.44 & $(3.30,46.97)$ \\
\hline & No & 2 & 10 & 12 & 1.00 & NA \\
\hline Wild anımal exposure & Yes & 28 & 26 & 54 & 5.39 & $(1.08,26.92)$ \\
\hline
\end{tabular}

each scale. At LSI $1(0.5 \mathrm{~km})$, the best model consisted of a single factor in hydrographic density $(P=0.0017$; ROC AUC $=0.71)$, which was positively correlated with infection. The best model for LSI $2(2 \mathrm{~km})$ included urban cover and agriculture $(P=0.05$; ROC AUC $=0.63)$; these factors were negatively correlated with infection. For LSI $3(5 \mathrm{~km})$ the best model contained solely percent agriculture $(P=0.08$; ROC AUC $=0.57)$, which was again negatively correlated with infection. Lastly, at LSI $4(10 \mathrm{~km})$, the best model contained percent open space, percent wetlands, and percent agriculture $(P=$ 0.02; ROC AUC $=0.67$ ); only agriculture had a negative parameter estimate, while open space and wetlands were positively correlated with infection. From this approach, we concluded that localized effects of LSI 1 constructed the best single scale model based on ROC values, albeit weakly.

To gain a more synoptic view of the effect of landscape parameters on observed infections, we constructed a combined scale model with factors from multiple LSI. Again using forward stepwise nominal logistic regression $(P<0.10)$, we found that the best combined model $(P<0.0001$; ROC AUC $=0.85)$ included five factors from four scales. At LSI 1, hydrographic density was positively correlated with infection $\left(\mathrm{LR} \chi^{2}=18.94\right.$; $P<0.0001$; OR $=375.15$; $95 \%$ CI $19.88-$ 13 303), whereas agriculture was negatively associated with infection at LSI 2 $\left(\mathrm{LR} \chi^{2}=5.77 ; P=0.0163 ;\right.$ OR $=0.0006$; $95 \%$ CI $<0.0001-0.37)$. At LSI 3, percent wetland was a positive factor (LR $\chi^{2}=8.21 ; P=0.0042 ;$ OR $37.35 ; 95 \%$ CI 3.03-1 221). At LSI 4, percent open space factored positive (LR $\chi^{2}=11.19 ; P=$ 0.0008 ; OR $=6189.29 ; 95 \%$ CI 23.36$>1000000$ ), while percent agriculture was negative in predicting cases $\left(\mathrm{LR} \chi^{2}=4.15\right.$; $P=0.0416$; OR 0.12 ; $95 \%$ CI $0.0088-$ $0.93)$.

\section{DISCUSSION}

Adin and Cowgill [1] described 36 cases of canine leptospirosis from California that were seen at the VMTH between 1990 and 1998 , with $30(83 \%)$ of these cases 
occurring in the last three years (19961998). We identified 43 cases in a threeyear period (1/1/1998 to $12 / 31 / 2000)$, suggesting an increase in the incidence of canine leptospirosis since 1996. We cannot completely rule out diagnostic bias, but the VMTH has performed active dog leptospirosis surveillance since at least $1990^{2}$. However, recent national diagnostic data also indicate that there is an increase in incidence of canine leptospirosis in the USA [14]. The most frequent serovar identified in dogs from northern California was pomona $(39.5 \%)$, similar to the $44 \%$ previously reported [1]. In the Midwestern USA, serovar grippotyphosa was the main serovar identified in cases of leptospirosis in dogs $(72.1 \%$ in a study from Illinois and $59 \%$ in a study from Indiana) $[4,23]$, whereas it was rather uncommon in northern California ( $<5 \%$ of the cases). Such a difference in serovar prevalence is most likely due to the presence of different reservoir species. While there has not been any recent work in California on identifying wildlife reservoirs of leptospirosis, four of 30 skunks tested in Connecticut had serologic evidence of infection with serovar grippotyphosa [17]. While none of the skunks in this study had evidence of infection with serovar pomona, previous research has shown that skunks could also be reservoirs of serovar pomona $[17,20]$. Richardson and Gauthier [17] also found that 11 of 31 raccoons had serologic evidence of infection with serovar icterohaemorrhagiae. One factor that may explain the preponderance of serovar pomona in California could be the cattle industry. In one recent study from Texas, 263 out of $1193(22.3 \%)$ cattle had at least a titer of 1:100 against serovar pomona [19]. With the large number of cattle present in California, it is likely that serovar pomona is common in the environment, and may

\footnotetext{
${ }^{2}$ Cowgill L., personal communication.
}

be carried by skunks, raccoons, and other small mammals.

Our results also included four cases of leptospirosis caused by serovar canicola and two cases caused by serovar icterohaemorrhagiae. These results are important because leptospirosis due to these two serovars has rarely been diagnosed in the last 10 years $[1,16,25]$. In recent years, portions of the general public have declined using the leptospirosis vaccine in their dogs due to a perceived risk of vaccine reaction and a lesser risk of infection with leptospirosis. The increased incidence of infection with serovars canicola and icterohaemorrhagiae may indicate that the lack of leptospirosis vaccination in the general dog population has influenced the incidence of these serovars as well. However, it is difficult to assess the significance of these cases since there were only six cases reported and our study was not designed to look at changes in incidence over time. It is important for pet owners and veterinarians to realize that a lack of compliance in using the Leptospira portion of the standard canine vaccine may lead to increased incidence of leptospirosis infections with serovars icterohaemorrhagiae and canicola.

Several recent studies have examined the demographic characteristics of Leptospira infected dogs, but the findings are ambiguous. Rentko et al. [16] did not find any age or breed predilection, but found more infected males than females. Harkin and Gartrell [9] found more infected females $(n=10)$ than infected males $(n=$ 7 ), and almost half of their cases were either German shepherds or German shepherd mixed-breed dogs $(n=8)$. Ward et al. [22] found that sexually intact males had a greater risk than sexually intact females, and also found that herding dogs, working dogs, and hounds had a greater risk than companion dogs. Age was also significantly associated with risk of leptospirosis, since dogs that were four to nine 
years old had significantly greater odds of contracting leptospirosis than dogs less than one year old [22]. Our logistic regression analysis found that there were no significant breed differences. This finding may have been due to the small numbers of cases and controls. We found a different age group at risk than reported by Ward et al. [22]. The clinical significance of our results could be questionable because of our small sample size, but may be due to the behavior and naïve immune status of younger animals and the weakened immune status of older animals. One explanation for this difference is the geographical origin of the cases and regional differences as it relates to sources of infection.

The county of residence (coastal versus inland counties) was marginally significant. While coastal waters are not directly implicated in the disease, this factor correlates with coastal regions having higher rainfall and more standing water, which facilitate increased Leptospira survival [7]. Previous studies have shown that canine leptospirosis is seasonal and that there is a significant correlation between cases of leptospirosis and rainfall $[1,21]$. In the San Francisco bay area, annual rainfall was positively correlated with the incidence of canine leptospirosis [1]. However, our results did not show a positive correlation with rainfall, possibly due to the small temporal and spatial scales of our study.

Although many environmental variables are important in the ecology of leptospirosis, our goal was to specifically identify some of these risk factors and to further quantify their importance. The unconditional logistic regression model identified five significant risk factors. It is unclear why dogs in one residence versus those of two residences have such greater odds of being infected with leptospirosis. It is possible that dogs in only one residence had more consistent exposure and therefore a greater chance of infection. We included this question to look at the possibility that dogs with two residences may spend a large amount of time at a lake or mountain home but could not draw a conclusion from the data.

Rural environments are thought to pose a higher risk of infection because these environments tend to have larger numbers of livestock, rodents, and small mammals, which are typical reservoirs for Leptospira [17, 24]. Rural environments also tend to have more standing water, such as irrigation canals and reservoirs. Water plays a large role in the ecology of Leptospira and is generally necessary for the occurrence of any epidemic. We could not identify any specific water source as a higher risk factor, but the primary risk factors for infection with leptospirosis were swimming or drinking from an outdoor water source, with respective odds ratios of eight and 12.44. Indirect exposure to wild animals, as reported by owners, also posed a significant risk factor for infection in the case-control study.

Based on our results as well as the work of Ward et al. [24], we believe that habitats where there are a large number of interactions between canines and wildlife pose a significant risk for acquiring leptospirosis. Ward et al. [24] also used GIS to identify environmental risk factors and reported that being located within $1000 \mathrm{~m}$ of an area that was urbanized between 1990 and 2000 was significant for increased risk of developing leptospirosis. While our study did not consistently find urbanization to be a significant factor of influence, we did find that surrogates for wildlife reservoir exposure (e.g. open space and wetlands) were important factors.

The relationship between disease status and the distance to the nearest hydrographic feature was apparent from our study. Hydrographic density is measured as unit length of a water feature per unit area of the LSI; thus it indirectly measures potential dog-to-water contact within that 
LSI. At close proximity, the important factor probably was exposure to leptospires and not the amount of habitat available for supporting leptospires. One of the interesting features of this analysis is the small amount of variation in case outcome explained by the distance to the nearest hydrographic feature, despite the highly significant relationship that existed. A possible explanation for the small amount of variation determined by geographic variables is the individual behavior of the dogs themselves. Different breeds of dogs, and even individuals within the same breed, can show marked differences in their attraction to water, and therefore their risk of exposure to leptospirosis.

Our results show that the best logistic regression model for a single LSI is the model for LSI 1, where hydrographic density is positively correlated with infection. In the logistic regression model for LSI 2 urban cover and agriculture are both negatively correlated with infection. Our definition of agriculture is an area where $>75 \%$ of the land is planted. So both agricultural land and urban cover would have almost no standing water, would not provide a good habitat for most small mammals, and are therefore not ideal for the transmission of leptospirosis. For LSI 3 agriculture is again negatively correlated with infection.

The second best logistic regression model for a single LSI is the model for LSI 4, where open space and wetlands are positively correlated with infection, and agriculture is again negatively correlated with infection. A likely reason that public open space becomes significant at LSI 4 (radius $=10 \mathrm{~km}$ ) is that there is a minimum number of reservoir hosts needed for an infectious disease to maintain itself in a specific environment, termed Critical Community Size [11]. Large tracts of public open space are more likely to contain large numbers of raccoons, opossums, and skunks, and lead to higher risk of exposure to leptospirosis. Large tracts of open space with higher amounts of wetlands are ideal habitats for the presence of leptospires and their reservoirs.

When we looked at combined scale models we found that the best combined model is one where hydrographic density is positively correlated in LSI 1, percent agriculture is negatively correlated in LSI 2, percent wetlands is positively correlated in LSI 3, and percent open space is positively correlated while percent agriculture is negatively correlated in LSI 4, reinforcing the findings of the individual LSI models.

One aspect of retrospective case-control studies that could affect the results of our study is recall bias. The general concern with these situations is that owners of sick dogs will recall the past better than the individuals associated with control dogs. There are many proposed reasons for this bias but the primary reasons are believed to be an extensive search for the cause of disease, and better recall associated with cases due to the stress of the situation. While we cannot completely rule out recall bias it is unlikely to affect our results because our controls were all leptospirosis suspects at one point, so the same search for explanations could have taken place. Another possible bias of our study was our selection of controls, which were dogs that were suspected of having leptospirosis but were seronegative. Generally these are animals that are referred to the VMTH with acute renal failure. There are many causes of acute renal failure in dogs, but the majority of cases of acute renal failure in dogs are caused by toxicities. While we cannot rule out any bias with our selection of controls, causes of toxicities are generally iatrogenic and randomly distributed in space with relation to the natural environment.

One of the limitations of this study is that we were not able to identify and count potential wildlife reservoirs of leptospirosis, and directly quantify the levels of risk in different habitats. Another limitation of 
this study was that it was not possible to isolate the infecting Leptospira serovars and in this way specifically define which risk factors apply to each of the serovars. Future research should focus on isolating Leptospira organisms from water sources and on identifying the specific reservoir species involved in canine leptospirosis infections, with emphasis placed on skunks in California. The GIS study's results validate the findings of the case-control study and underline the usefulness of GIS in elucidating environmental risk factors for infectious diseases. While it was important to demonstrate the correlation between the results of the case-control study and the GIS analysis, it is now clear that GIS analysis of possible risk factors can be used alone. Overall, we have demonstrated that GIS analysis in conjunction with more traditional epidemiological analysis can be a powerful tool in identifying risk factors for infectious diseases.

\section{ACKNOWLEDGEMENTS}

This study was funded by generous support from The Center for Companion Animal Health through a special grant provided by Mary Ann Charles. I would like to thank the staff at The Center for Companion Animal Health, Rickie Kasten, Amy Poland, and Dr Janet Foley.

\section{REFERENCES}

[1] Adin C.A., Cowgill L.D., Treatment and outcome of dogs with leptospirosis: 36 cases (1990-1998), J. Am. Vet. Med. Assoc. (2000) 216:3371-3375.

[2] Beardsley K., Stoms D., Compiling a Digital Map of Areas Managed for Biodiversity in California, Natural Areas J. (1993) 13:177190.

[3] Bolin C.A., Diagnosis of leptospirosis: a reemerging disease of companion animals, Sem. Vet. Med. Surg. (Small Anim.) (1996) 11:166-171.
[4] Boutilier P., Carr A., Schulman R.L., Leptospirosis in dogs: a serologic survey and case series 1996 to 2001, Vet. Ther. (2003) 4:178-187.

[5] Cowardin L.M., Wetland Classification in United-States, J. For. (1978) 76:666-668.

[6] Daly C., Neilson R.P., Phillips D.L., A statistical topographic model for mapping climatological precipitation over mountainous terrain, J. Appl. Meteorol. (1994) 33:140 158.

[7] Faine S., Leptospira and leptospirosis, CRC Press, Boca Raton, 1994.

[8] Farr R.W., Leptospirosis, Clin. Infect. Dis. (1995) 21:1-20.

[9] Harkin K.R., Gartrell C.L., Canine leptospirosis in New Jersey and Michigan: 17 cases (1990-1995), J. Am. Anim. Hosp. Assoc. (2002) 56:203-213.

[10] Homer C., Huang C.Q., Yang L.M., Wylie B., Coan. M., Development of a 2001 National Land-Cover Database for the United States, Photogramm. Eng. Remote Sensing (2004) 70:829-840.

[11] Kermack W.O., McKendrick A.G., A contribution to the mathematical theory of epidemics, Proc. R. Soc. Lond. (1927) 115:700 721.

[12] Maupin G.O., Fish D., Zultowski J., Campos E.G., Piesman J., Landscape ecology of Lyme disease in a residential area of Westchester County, New York, Am. J. Epidemiol. (1991) 133:1105-1113.

[13] Menard S., Applied Logistic Regression Analysis, Sage University Press, Thousand Oaks, 1995.

[14] Moore G.E., Guptill L.F., Glickman N.W., Caldanaro R.J., Aucoin D., Glickman L.T., Canine leptospirosis, United States, 20022004, Emerg. Infect. Dis. (2006) 12:501503.

[15] Reisen W.K., Lothrop H.D., Presser S.B., Hardy J.L., Gordon E.W., Landscape ecology of arboviruses in southeastern California: temporal and spatial patterns of enzootic activity in Imperial Valley, 1991-1994, J. Med. Entomol. (1997) 34:179-188.

[16] Rentko V.T., Clark N., Ross L.A., Schelling S., Canine leptospirosis: A retrospective study of 17 cases, J. Vet. Intern. Med. (1992) 6:235-244. 
[17] Richardson D.J., Gauthier J.L., A serosurvey of leptospirosis in Connecticut peridomestic wildlife, Vector Borne Zoonotic Dis. (2003) 3:187-193.

[18] Swets J.A., Pickett R.M., Evaluation of diagnostic systems: methods from signal detection theory, Academic Press, New York, 1982.

[19] Talpada M.D., Garvey N., Sprowls R., Eugster A.K., Vinetz J.M., Prevalence of leptospiral infection in Texas cattle: implications for transmission to humans, Vector Borne Zoonotic Dis. (2003) 3:141-147.

[20] Verts B.J., The biology of the striped skunk, University of Illinois Press, Baltimore, Maryland, 1964.

[21] Ward M.P., Seasonality of canine leptospirosis in the United States and
Canada and its association with rainfall, Prev. Vet. Med. (2002) 56:203-213.

[22] Ward M.P., Glickman L.T.G., Guptill L., Prevalence of and risk factors for leptospirosis among dogs in the United States and Canada, J. Am. Vet. Med. Assoc. (2002) 220:53-58.

[23] Ward M.P., Guptill L.F., Prahl A., Wu C.C., Serovar-specific prevalence and risk factors for leptospirosis among dogs: 90 cases (1997-2002), J. Am. Vet. Med. Assoc. (2004) 224:1958-1963.

[24] Ward M.P., Guptill L.F., Wu C.C., Evaluation of environmental risk factors for leptospirosis in dogs: 36 cases (1997-2002), J. Am. Vet. Med. Assoc. (2004) 225:72-77.

[25] Wohl J.S., Canine leptospirosis, Comp. Small Anim. (1996) 18:1215-1224. 